Memoirs of The College of Science, University of Kyoto, Sesirs A

Vol. XXVIII, Mathematics No. 2, 1953.

\title{
Note on integral closures of Noetherian domains
}

By

\author{
Masayoshi Nagata
}

(Received November 3, 1953)

Previously Prof. Akizuki" proved that if $\mathfrak{o}$ is a Noetherian local integrity domain ${ }^{2)}$ of dimension 1 and if $\hat{v}$ is its integral closure ${ }^{3)}$, then any ring $\mathfrak{z}$ such that $\mathfrak{v} \subseteq \mathfrak{g} \subseteq \hat{\mathfrak{v}}$ is Notherian ${ }^{4)}$.

As for the case of higher dimension, there arise the following problems :

Let $\mathfrak{o}$ be a Noetherian local integrity domain of dimension $n$ and let $\hat{\mathfrak{o}}$ be its integral closure. Then

Problem I. Does it holds in general that any ring $\mathfrak{z}$ such that $\mathfrak{v} \subseteq \mathfrak{g} \subseteq \hat{\mathfrak{v}}$ is Noetherian?

Problem II. Does it holds in general that $\hat{\mathfrak{v}}$ is Noetherian?

In the present note, we show a counter example against the problem I when $n=2$ in $\S 2$ and then a counter example against the problem II when $n=3$ in $\S 3^{6)}$.

\section{$\S 1$. A preliminary.}

Let $\mathfrak{f}_{0}$ be a perfect field of characteristic $p(\neq 0)$ and let $u_{1} \cdots$, $u_{n}, \cdots$ (infinitely many) be algebraically independent elements over $\mathfrak{f}_{0}$. Set $\mathfrak{f}=\mathfrak{f}_{0}\left(u_{1}, \cdots, u_{n}, \cdots\right)$. Further let $x_{1}, \cdots, x_{n}$ be indeterminates and denote by $\mathfrak{o}_{n}$ and $\mathfrak{r}_{n}$ the rings $\mathfrak{f}^{p}\left\{x_{1}, \cdots, x_{n}\right\}[\mathfrak{f}]$ and $\mathfrak{f}\left\{x_{1}, \cdots, x_{n}\right\}^{\left({ }^{3)}\right.}$ respectively.

1) Y. Akizuki, Einige Bemerkunge über primäre Integritätsbereiche mit Teilerkettensatz, Proc. Phys.-Math. Soc. Japan, 3rd Ser., 17 (1935), pp. 327-336.

2) We say in the present note that a ring $o$ is a local ring if it has only one maximal ideal $m$ and if the intersection of all powers of $m$ is zero, where we consider the m-adic topology for $\mathbf{D}$.

3) This means the integral closure in its quotient field.

4) This result shows also the similar result for " einartig" Noetherian integrity domains.

5) It was communicated to the writer that this problem II was proved affirmatively by $\mathrm{Mr}$. Mori, when $n=2$.

6) $\left\{\left\{x_{1}, \cdots, x_{n}\right\}\right.$ denotes the ring of formal power series in $x_{1}, \cdots x_{n}$ with coefficients in $t$. 
Then we have

Lemma. $\mathfrak{o}_{n}$ is a regular local ring and $\mathfrak{r}_{n}$ is the completion of $\mathfrak{D}_{n}$.

Proof. When we see that $\mathfrak{b}_{n}$ is Noetherian, our assertion follows easily. Therefore we prove that $\mathfrak{o}_{n}$ is Noetherian. When $n=1$, our assertion is evident. Therefore we prove our assertion by induction on $n$. Since $\mathfrak{f}^{p}\left\{x_{1}, \cdots, x_{n}\right\}$ is a complete regular local ring, $\mathfrak{f}^{n}\left\{x_{1}, \cdots, x_{n}\right\}\left[a_{1}, \cdots, a_{t}\right]$ is a complete regular local ring and therefore it is an integrally closed integrity domain, provided that $a_{i}$ $\epsilon$. This shows that $\mathfrak{o}_{n}$ is integrally closed. Therefore if $y$ is an element of $\mathfrak{o}_{n}, y \mathfrak{r}_{n} \cap \mathfrak{o}_{n}=y \mathfrak{o}_{n}{ }^{i}$. Now let $q$ be an arbitrary prime ideal of $\mathfrak{o}_{n}$; we have only to show that $\mathfrak{q}$ has a finite basis ${ }^{8)}$. Let $\mathfrak{p}$ be a minimal prime ideal of $\mathfrak{o}_{n}$ contained in $\mathfrak{q}$ and let $\bar{p}$ be the prime ideal of $\mathfrak{r}_{n}$ such that $\overline{\mathfrak{p}} \cap \mathfrak{o}_{n}=\mathfrak{p}$. Set $\mathfrak{v}_{0}=\mathfrak{f}^{p}\left\{\boldsymbol{x}_{1}, \cdots, x_{n}\right\}$ and $\mathfrak{p}_{0}=$ $\mathfrak{p} \cap \mathfrak{o}_{0}$. Then since $\mathfrak{o}_{0}$ is regular, we see that $\mathfrak{p}_{0}$ is a principal ideal : $\mathfrak{p}_{0}=f \mathfrak{o}_{0}$. Since $\mathfrak{o}_{0}$ is complete, we may assume that $f=a_{0}+a_{1} x_{n}+\cdots$ $a_{s-1} x_{n}^{s-1}+x_{n}^{s}$ with $a_{i} \in \mathfrak{D}^{\prime}=\mathfrak{f}^{p}\left\{x_{1}, \cdots, x_{n-1}\right\}$ by Weierstrass preparation theorem.

Case 1). When $f$ is irreducible over $\mathfrak{o}_{n-1}$, we see that $f \mathfrak{o}_{n}$ is prime because $\mathfrak{o}_{n-1}$ is integrally closed. Hence $\mathfrak{p}=\boldsymbol{f}_{\mathfrak{o}_{n}}$.

Case 2). When $f$ is not irreducible over $\mathfrak{o}_{n-1}$, we take an irreducible monic factor $f^{\prime}$ of $f$ in the polynomial ring $\mathfrak{v}_{n-i}\left[x_{n}\right]$. Since $\mathfrak{o}_{n-1}$ is a purely inseparable integral extension of $\mathfrak{v}^{\prime}$ with exponent $p, f=f^{\prime \prime}$. Therefore $f^{\prime} \mathfrak{r}_{n}$ must be a prime ideal, because $\mathfrak{r}_{n}$ is a purely inseparable integral extenson of $\mathfrak{v}_{0}$ with exponent $p$. Hence $f^{\prime} \mathfrak{o}_{n}=f^{\prime} \mathfrak{r}_{n} \cap \mathfrak{o}_{n}$ is a prime ideal.

Thus, in either case, we see that $\mathfrak{p}$ is principal and that $\mathfrak{o}_{n} / \mathfrak{p}$ is a finite module over $\mathfrak{v}_{n-1}$ (and therefore that $\mathfrak{o}_{n} / \mathfrak{p}$ is Noetherian). This shows that $\mathfrak{q}$ has a finite basis.

\section{$\S 2$. A counter example against the problem $I$.}

We denote, in this paragraph, by $x$ and $y$ instead of $x_{1}$ and $x_{2}$ respectively. We take elements $c=y \sum_{i=1}^{\infty} u_{i} x^{i}, c_{n}=\left(c-\sum_{i<n} y u_{i} x^{i}\right) / x^{n}$ $(n=1,2, \cdots)$. We consider the ring $\mathfrak{v}=\mathfrak{v}_{2}\left[c_{1}, \cdots, c_{n}, \cdots\right]$.

Proposition $1 . \quad \mathfrak{o}$ is a counter example against the problem I.

7) Observe that $r_{n}$ is integral over $o_{n}$.

8) Cf. I. S. Cohen, Commutative rings with restricted minimum condition, Duke Math. J., 17 (1950), pp. 27-42. 
Proof. Since $\mathfrak{o}_{2}$ is Noetherian, $\mathfrak{o}_{2}[c]$ is Noetherian. It is evident that $\mathfrak{o}$ contains $\mathfrak{v}_{2}[c]$ and is contained in the integral closure of $\mathfrak{v}_{2}[c]$. Therefore we have only to show that $\mathfrak{v}$ is not Noethrian.

We first show that this local ring $\mathfrak{v}$ is a dense subspace of $\mathfrak{r}_{\mathfrak{2}}$. Let $b$ be an element of $(x, y)^{r} r_{2} \cap \mathfrak{v}$. Since $b$ is in $\mathfrak{v}$, we can write $b$ as a polynomial in $c_{1}, \cdots, c_{n}$ (by a suitable $n$ ) with coefficients in $\mathfrak{0}_{2}$. Since $c_{i}=u_{i}+x c_{i+1}$, we can write $b$ as a polynomial in $x^{r} c_{n+r}$ with coefficients in $\mathfrak{v}_{2}: \quad b=b_{0}+b_{1}\left(x^{r} c_{n+r}\right)+\cdots+b_{s}\left(x^{r} c_{n+r}\right)^{s}\left(b_{i} \in \mathfrak{D}_{2}\right)$. Since $b \in(x, y)^{r} \mathfrak{r}_{2}$, we have $b_{0} \in(x, y)^{r} \mathfrak{r}_{2} \cap \mathfrak{v}_{2}=(x, y)^{\prime} \mathfrak{v}_{2} \subseteq(x, y)^{r}$. Therefore $b \in(x, y)^{r} \mathfrak{v}$, which shows that $(x, y)^{r} \mathfrak{r}_{2} \cap \mathfrak{v}=(x, y)^{r} \mathfrak{v}$. Now that $\mathfrak{v}$ is dense in $\mathfrak{r}_{2}$ is evident. We see that the completion of $\mathfrak{v}$ is regular. Therefore, if $\mathfrak{v}$ is Noetherian, $\mathfrak{v}$ must be regular and therefore $\mathfrak{v}$ must be integrally closed. But $\mathfrak{v}$ cannot be integrally closed because $c_{1} / y$ is not in $\mathfrak{v}$.

\section{$\$ 3$. A counter example against the problem II.}

We denote, in this paragraph, by $x, y$ and $z$ instead of $x_{1}, x_{2}$ and $x_{3}$ respectively. Further we denote by $u_{1}, v_{1}, u_{2}, v_{2}, \cdots$ instead of $u_{1}, u_{2} u_{3} u_{4}, \cdots$ respectively. Take an element $c=y \sum_{i=1}^{\infty} u_{i} x^{i}+z \sum_{i=1}^{\infty} v_{i} x^{i}$ of $\mathfrak{r}_{3}$ and let $\mathfrak{v}$ be the integral closure of $\mathfrak{v}_{3}[c]$. For the simplicity of our calculus, we treat the case $p=2^{9}$.

Proposition 2. $\mathfrak{v}_{3}[c]$ is a counter example against the problem II.

Proof. That $\mathfrak{o}_{3}[c]$ is Noetherian is evident. Therefore we have only to show that $\mathfrak{o}$ is not Noetherian. Since $\mathfrak{v}$ is integrally closed, $x \mathfrak{r}_{3} \cap \mathfrak{v}=x \mathfrak{v}$ and therefore $x \mathfrak{v}$ is a prime ideal. We consider valuation rings $\mathfrak{v}^{\prime}=\mathfrak{v}_{\left(x_{\left.\mathfrak{v}_{3}\right)}\right)}$ and $\mathfrak{v}^{\prime \prime}=\mathfrak{v}\left(x_{\mathfrak{v}}\right)$. Then since $c$ is in the completion of $\mathfrak{v}^{\prime}$, we see that $\mathfrak{v}^{\prime}$ is a dense subspace of $\mathfrak{v}^{\prime \prime}$ and therefore $\mathfrak{v}^{\prime} / x \mathfrak{v}^{\prime}=\mathfrak{v}^{\prime \prime} / x \mathfrak{v}^{\prime \prime}$. On the other hand, since $\mathfrak{v}_{3} / x \mathfrak{v}_{3}$ is regular, $\mathfrak{v}_{3} / x \mathfrak{v}_{3}$ is integrally closed. Therefore $\mathfrak{v}_{3} / x \mathfrak{v}_{3}=\mathfrak{v} / x \mathfrak{v}$. Therefore the maximal ideal of $\mathfrak{v}$ can be generated by $x, y$ and $z$. Therefore, if $\mathfrak{v}$ is Noetherian, $\mathfrak{v}$ must be regular. Now we have only to show that $\mathfrak{v}$ is not regular.

Assume for a moment that $v$ is regular. Then $\mathfrak{v}$ must contain

9) We need not for our calculus that $p=2$. Whenever $p$ is not equal to zero, the same construction yields a counter example against our:problem II. Our calculus for the case is similar, but it is somewhat more complicated, because there must appear some more terms in the formulars below. 
an element $\sum_{i=1}^{\infty} u_{i} x^{i}+z f$ with a suitable $f \in \mathfrak{r}_{3}$ because $\mathfrak{o} / 2 \mathfrak{v}$ is regular. Therefore we can write

$$
\sum_{i=1}^{\infty} u_{i} x^{i}+z f=\left(a_{0}+a_{1} c\right) / d_{1} \quad\left(a_{0}, a_{1}, d_{1} \in \mathfrak{O}_{3}\right),
$$

where we choose $a_{0}, a_{1}, d_{1}$ so that they have no common factor. Now we have

$$
d_{1} \sum_{i=1}^{\infty} u_{i} x^{i}+d_{1} z f=a_{0}+a_{1} y \sum_{i=1}^{\infty} u_{i} x^{i}+a_{1} z \sum_{i=1}^{\infty} v_{i} x^{i} .
$$

Since 1, $\sum u_{i} x^{i}$ are linearly independent over $\mathfrak{v}_{2}\left(=v_{3} / z \mathfrak{v}_{3}\right)$, we have $a_{0} \in z \mathfrak{v}_{3}, d_{1}-a_{1} y \in z \mathfrak{0}_{3}$. Therefore we can write $a_{0}=z a_{0}{ }^{\prime}, d_{1}=a_{1} y+d z$ $\left(a_{0}^{\prime}, d \in \mathfrak{D}_{3}\right)$. Then

$$
\left(a_{1} y+d z\right)\left(\sum u_{i} x^{i}+f_{2}\right)=z a_{0}^{\prime}+a_{1} y \sum u_{i} x^{i}+a_{1} z \sum v_{i} x^{i},
$$

and therefore

$$
a_{1} y z f+d z \sum u_{i} x^{i}+d f z^{2}=z a_{0}{ }^{\prime}+a_{1} z \sum v_{i} x^{2} .
$$

we write $a_{1}=\sum_{i=0}^{\infty} a_{18} z^{i}, a_{0}^{\prime}=\sum_{i=0}^{\infty} a_{01} z^{i}, d=\sum_{i=0}^{\infty} d_{i} z^{i}, f=\sum_{i=0}^{\infty} f_{i} z^{i} \quad\left(a_{j i}, d_{i} \in \mathfrak{D}_{3}\right.$, $\left.f_{i} \in r_{2}\right)$. Then comparing the coefficients of $z$, we have

$$
a_{10} y f_{0}+d_{0} \sum u_{i} x^{i}=a_{00}+a_{10} \sum v_{i} x^{i} .
$$

Since 1, $\sum u_{i} x^{i}$ and $\sum v_{i} x^{i}$ are linearly independent over $\mathfrak{v}_{1}\left(=\mathfrak{o}_{2} / y \mathfrak{o}_{2}\right)$, we have $d_{0}, a_{00}$ and $a_{10}$ are in $y_{0}$. We show next that $d_{r}, a_{0 r}$ and $a_{1}$. are in $y 0_{2}$ for any $r$, by induction on $r$. Comparing the coefficients of $z^{r+1}$, we have

$$
y\left(\sum_{j=0}^{r} a_{1 j} f_{r_{j}}\right)+d_{r} \sum u_{i} x^{i}+\sum_{j=0}^{n-1} d_{j} f_{r-1-j}=a_{0,}+a_{1}, \sum v, x^{i} .
$$

Since $d_{0}, d_{1}, \cdots, d_{r-1}$ are in $y v_{2}$, by our induction assumption, we have that $d_{r}, a_{0 r}$ and $a_{1,}$ are in $y_{0 .}$.

Thus we see that $d_{1}, a_{11}$ and $a_{1}$ are in $y 0_{3}$, which is a contradiction to that $\boldsymbol{d}_{\mathrm{i}}, a_{0}$ and $a_{1}$ have no common factor. Thus our proof is completed. 